\title{
Ablation Compared to Drug Therapy for Recurrent Ventricular Tachycardia in Arrhythmogenic Right Ventricular Cardiomyopathy; Results from a Multicenter Study
}

Saagar Mahida ${ }^{1,2}$, MBChB, PhD, Jeroen Venlet ${ }^{3}$, MD, Ardan Muammer Saguner ${ }^{4}$, MD, Saurabh Kumar ${ }^{1,5}$, BSc(Med)/MBBS, PhD, Samuel H Baldinger ${ }^{1,6}, \mathrm{MD}$, Amir AbdelWahab7 ${ }^{7}, \mathrm{MD}$, Usha B. Tedrow ${ }^{1}, \mathrm{MD}$, Silvia Castelletti ${ }^{8}, \mathrm{MD}$, Antonis Pantazis ${ }^{9}$, MD, Roy M John ${ }^{1}$ MD, PhD, William J McKenna ${ }^{9}, M D$, Pier D. Lambiase ${ }^{9}, M D$, Firat Duru $^{4}$ MD, John L Sapp ${ }^{7}$, MD, Katja Zeppenfeld ${ }^{3}$, MD, and William G. Stevenson ${ }^{1}, M D$

1. Arrhythmia Unit, Cardiovascular Division, Brigham and Women's Hospital, Boston. MA. USA.

2. Department of Cardiac Electrophysiology, Liverpool Heart and Chest Hospital, UK

3. Leiden University Medical Center, Department of Cardiology, Leiden, The Netherlands

4. Department of Cardiology, University Heart Center Zurich, Zurich, Switzerland

5. Cardiology Department, Westmead Hospital, Westmead, Sydney, NSW, Australia

6. Department of Cardiology, Inselspital, Bern University Hospital, and University of Bern, Switzerland

7. Division of Cardiology, Queen Elizabeth II Health Sciences Centre, Halifax, Nova Scotia, Canada

8. Center for Cardiac Arrhythmias of Genetic Origin, Istituto Auxologico Italiano "San Carlo", Milan, Italy

9. Department of Cardiac Electrophysiology, The Heart Hospital, Institute of Cardiovascular Science, University College London, London, UK 
Running title: ARVC and VT ablation

Word count: 1,769

Acknowledgements: PL is supported by the UCLH and Bart's Biomedicine NIHR.

Conflicts of interest: None declared

\section{Corresponding author:}

Dr. William G. Stevenson.

Cardiovascular Division.

Brigham and Women's Hospital.

75 Francis St. Boston. MA. 02115.

Email:wstevenson@partners.org. 


\begin{abstract}
Background: The comparative efficacy of antiarrhythmic drug therapy (AAD) versus ventricular tachycardia (VT) ablation in arrhythmogenic right ventricular cardiomyopathy (ARVC) is unknown.
\end{abstract}

Objectives: We compared outcomes of AAD and/or beta blocker (BB) therapy to VT ablation (with $\mathrm{AAD} / \mathrm{BB}$ ) in ARVC patients with recurrent VT.

Methods: In a multicenter retrospective study, 110 ARVC patients (38 17 years, 83\% male) with a minimum of 3 VT episodes were included; 77 (70\%) were initially treated with AAD/BB and 32 (29\%) underwent ablation. Subsequently, 43 of the 77 patients treated with AAD/BB-only also underwent ablation. Overall, 75 patients underwent ablation.

Results: When comparing initial AAD/BB therapy $(n=77)$ and VT ablation $(n=32)$ after $\geqq 3$ VT episodes, a single ablation procedure rendered $35 \%$ of patients free of VT at 3 years compared to $28 \%$ of $A A D / B B-o n l y$ treated patients $(p=0.46)$. Of the 77 AAD/BB treated patients, 43 subsequently had ablation. For all 75 patients who had ablation, $56 \%$ were VT-free at 3 years after the last ablation. Epicardial ablation was used in 53\% and was associated with lower VT recurrence after the last ablation (endocardial/epicardial vs. endocardial-only; 71\% vs. 47\% three-year VT-free survival, $\mathrm{p}=0.05)$. Importantly, there was no difference in survival free of death or transplantation between the ablation- and AAD/BB-only treated patients $(p=0.61)$. 
Conclusion: Amongst ARVC patients with a high VT burden, mortality and transplantation-free survival is not significantly different between drug- and ablationtreated patients. These patients have a high risk of recurrent VT despite drug therapy. Combined endocardial/epicardial ablation is associated with reduced VT recurrence compared to endocardial-only ablation.

Keywords: Arrhythmogenic right ventricular cardiomyopathy, ventricular tachycardia, catheter ablation

Abstract word count: 250 


\section{Introduction}

2 Arrhythmogenic right ventricular cardiomyopathy (ARVC) is an inherited

3 cardiomyopathy characterized by fibrofatty replacement of myocardial tissue. ${ }^{1-4}$

$4 \quad$ ARVC is associated with an increased risk of ventricular tachycardia (VT) and sudden

5 cardiac death. ${ }^{5,6}$ Implantable cardiac defibrillators (ICD) have been reported to

6 improve long-term outcome in patients with ARVC and VT. ${ }^{7}$ In a subset of patients

7 with ICDs however, multiple shocks due to recurrent VT result in significant

8 morbidity. 8,9

9

10 Over the past two decades, a number of studies have reported that VT ablation can

11 reduce arrhythmia burden in ARVC, albeit with significant recurrence rates and a risk

12 of procedural complications. ${ }^{10-15}$ Catheter ablation has largely been reserved for

13 patients with a high ICD shock burden despite antiarrhythmic drug (AAD) or beta

14 blocker (BB) therapy, and the impact of adding ablation to AAD therapy relative to

15 continuing or escalating AAD therapy is not well studied. Furthermore, it is now clear

16 that for some patients epicardial ablation is more efficacious than endocardial

17 ablation, but may expose the patient to additional procedural risks. In view of these

18 considerations, the frequency of VT that warrants proceeding to catheter ablation is

19 uncertain. A recent trial in patients with post infarction VT found that patients with 3

20 or more episodes of VT or who had received an ICD shock despite antiarrhythmic drug

21 therapy had better composite outcomes with ablation rather than escalated

22 antiarrhythmic drug therapy. ${ }^{16}$ 
1 The aim of this multicenter study was to compare outcomes of these treatment

2 strategies in patients with ARVC who had recurrent VT ( $\geq 3$ episodes). All patients

3 were receiving $A A D$ and or $B B$ and we compared three groups: those who continued

4 to receive only drug therapy, those who received adjunctive therapy with endocardial

5 ablation, and those who received combined endocardial/epicardial ablation.

6

\section{$7 \quad$ Methods}

8

\section{Patient population}

10 Retrospectively identified ARVC patients were included from five tertiary cardiac

11 centers between January 2000 and May 2015. All patients fulfilled the 2010 Task

12 force criteria for a definite diagnosis of ARVC. ${ }^{17}$ Taskforce criteria were evaluated at

13 the time of inclusion into the study. An additional inclusion criterion was that all

14 patients experienced either 1 ) $\geq 3$ episodes of sustained VT (requiring either external

15 DC cardioversion, ATP, ICD shock, or acute chemical cardioversion) resulting in

16 separate presentations at distinct time points or, 2) $\geq 3$ cumulative appropriate shocks

17 for VT (either 3 consecutive shocks on the same presentation, i.e. VT storm, or 3

18 cumulative shocks over 2 separate presentations). The study was approved by the

19 Institutional Review Boards at the respective institutions.

20

\section{Electrophysiology study and catheter ablation}

22 The decision to treat with $\mathrm{AAD} / \mathrm{BB}$ or to perform $\mathrm{VT}$ ablation was at the treating

23 physician's discretion. In the subset of patients that underwent VT ablation,

24 endocardial mapping was performed in all patients. Epicardial mapping and ablation 
1 was performed in selected patients, also at the physicians' discretion. A percutaneous

2 subxyphoid approach was used to gain epicardial access. ${ }^{18}$ Three dimensional

3 electroanatomical substrate maps were created using either the Carto (Biosense

4 Webster, Diamond Bar, CA) or NavX (St. Jude Medical, St Paul, Minnesota) mapping

5 system. Normal bipolar voltage was defined as $>1.5 \mathrm{mV}$; scar was defined as $<0.5 \mathrm{mV}$;

6 and scar border zones were defined as 0.5-1.5 mV. ${ }^{19}$

7

8 All monomorphic VTs that the treating physician thought were clinically relevant

9 (based on cycle length/morphology matching clinically documented VT) were

10 targeted during ablation, including all mappable VTs. Conventional entrainment and

11 activation mapping techniques were used to identify critical target sites for mappable

12 VTs. A substrate-based approach was used in patients with unmappable VTs.

13 Substrate ablation targeted sites with low-amplitude electrograms with wide

14 fractionation (usually multicomponent electrograms $<0.5 \mathrm{mV} ;>133 \mathrm{~ms}$ ), sites with late

15 (usually $>10 \mathrm{~ms}$ after end of QRS) and split potentials (usually isoelectric period of

$16>30-50$ ms between spikes) and sites with a paced QRS morphology matching a VT

17 (usually with a stimulus to QRS interval of $>40 \mathrm{~ms}$ ). ${ }^{20,21}$ An example of a voltage map

18 and potential targets for substrate ablation are illustrated in Figure 1. VT inducibility

19 was assessed post-ablation with programmed stimulation using 3 extrastimuli (until

20 refractoriness or a minimum cycle length of 200 ms was reached).

21

22 Follow-up

23 The follow-up period for the comparison between AAD/BB therapy and VT ablation

24 began after the $3^{\text {rd }}$ VT episode/ $/ 3^{\text {rd }}$ shock. The follow-up period for the comparison 
1 between endocardial-only ablation and combined endocardial/epicardial ablation

2 began after the last VT ablation procedure. In patients with ICDs, data from sequential

3 ICD interrogations was documented. Failure of VT ablation or AAD/BB therapy was

4 defined as a recurrence of sustained VT, including episodes terminated by ICD shocks,

5 episodes treated with ATP, and monitored sustained VT episodes requiring DC

6 cardioversion or chemical cardioversion.

7

\section{Statistical analysis}

9 Data analysis was performed with SPSS version 23.0 (IBM SPSS, Armonk, NY).

10 Continuous variables were expressed as mean \pm standard deviation or median and

11 interquartile range (IQR). Continuous variables were compared using the Student's t-

12 Test or Mann-Whitney $U$ test. Categorical variables were compared using the $\chi^{2}$ test.

13 The endpoints of freedom from sustained VT and freedom from death/heart

14 transplantation were determined using Kaplan-Meier analysis. Only interventions

15 after three VT episodes/shocks were included in the analysis (failure of VT ablations

16 or AAD/BBs prior to three VT episodes were 'blanked').

18 Results

19 Patient population

20 The patient cohort comprised of 110 ARVC patients (specific numbers from each

21 contributing center are included in Supplemental table 1). Patient characteristics are

22 summarized in Table 1. The mean age at first presentation with a ventricular

23 arrhythmia was $38 \pm 17$ years. Patients were predominantly male (91[83\%]) and of

24 Caucasian descent (105 [95\%]). An ICD had been implanted in 109 (99\%) patients. 


\section{Antiarrhythmic drug therapy vs. single VT ablation procedure}

3 After $\geq 3$ VT episodes/3 shocks, 109/110 patients were treated with AAD/BB while one

4 patient had no therapeutic interventions. Of these 109 patients, 32 (29\%) underwent

5 an adjunctive ablation procedure (including epicardial ablation in 11 patients) after

6 the $3^{\text {rd }} V T$ episode $/ 3^{\text {rd }}$ shock. Numbers of patients undergoing ablation and AAD/BB

7 therapy are summarized in Figure 2 . The remaining 77 (71\%) were treated with

$8 \mathrm{AAD} / \mathrm{BB}$ alone (AAD/BB commenced in drug-naïve patients in 19 cases, AAD/BB

9 changed in 20, a second drug added to pre-existing therapy in 11, drug dose was

10 increased in 24, and no change in AAD in 3).

11

12 By 3 years, 35\% of the patients in the ablation group were free of VT after a single

13 ablation procedure while $28 \%$ of the patients in AAD/BB-only treated group were free

14 of $V T$ ( $p=0.46$, Figure 3$)$. When taking individual AAD into account, there were no

15 differences in outcome amongst patients treated with VT ablation and those treated

16 with amiodarone, sotalol, class 1 drugs, and beta blockers (Figure 4). Treatment with

17 amiodarone or class 1 agents was associated with a trend towards improved outcome

18 compared to sotalol therapy (amiodarone vs. sotalol $p=0.20$; class 1 vs. sotalol

$19 \mathrm{p}=0.12)$. When taking endocardial-only and combined endocardial/epicardial

20 ablations separately, there was a trend towards improved outcome in the combined

21 endocardial/epicardial group (endocardial-only vs. endocardial/epicardial ablation,

$22 p=0.19$; combined endocardial/epicardial group vs. $A A D, p=0.15$, Supplemental figure

$231)$. 


\section{$1 \quad$ Multiple ablation procedures}

2 Of the 77 patients treated initially with AAD/BB after $3 \mathrm{VT}$ recurrences, 43 underwent

3 ablation after more VT recurrences. Overall therefore, a total of 75 patients

4 underwent an ablation procedure (Figure 2). These patients had between 1 and 7

5 procedures (for patients who underwent $>1$ procedure, VT ablations were performed

6 over a period of $3.0 \pm 4.2$ years; range $0-17.5$ years); a single procedure in 37 [49\%]

7 patients; 2 procedures in 22 [29\%] patients; 3 procedures in 8 [11\%] patients; 4

8 procedures in 3 [4\%] patients; 5 procedures in 3 [4\%] patients; 6 procedures in 1 [1\%]

9 patient, and 7 procedures in 1 [1\%] patient. Forty of the 75 patients who underwent

10 ablation (53\%) had at least one combined endocardial/epicardial ablation procedure

11 and 35 (47\%) had exclusively endocardial ablations. Two patients had surgical VT

12 ablation procedures. The distribution of endocardial and combined

13 endocardial/epicardial ablation procedures for each contributing center is included in

\section{Supplemental figure 2.}

16 Follow-up data was available in 72 of the above 75 patients. By 3 years after the last

17 ablation procedure, $56 \%$ of patients were free of VT (Figure 5). When taking into

18 account combined endocardial/epicardial ablations separately, $71 \%$ of patients who

19 had at least one combined endocardial/epicardial procedure were free of VT at 3

20 years, compared to $47 \%$ of patients who exclusively underwent endocardial ablation

21 procedures ( $p=0.05$, Figure 6$)$. Amongst patients who experienced post ablation VT

22 recurrences, there was no significant difference in VT burden between the two

23 groups (endocardial-only vs. endocardial/epicardial ablation; VT episodes/shocks; 
1 median 2 [IQR 2.0; variance 11.1; skewness 2.46] vs. median 1 [IQR 2.5; variance 3.5;

2 skewness 8.80]; $p=0.86)$.

3

\section{Complications}

5 Procedure-related major complications occurred in 3 (4\%) patients (1 right ventricular

6 perforation, 1 myocardial infarction [ 14 days after epicardial ablation] ${ }^{22}$, and 1 case of

7 subclavian deep vein thrombosis following surgical ablation). There were no

8 procedure-related deaths.

9

\section{Survival following VT ablation and antiarrhythmic drug therapy}

11 Patients were followed-up for $6.1 \pm 4.5$ years after the $3^{\text {rd }} V T$ episode/ $/ 3^{\text {rd }}$ shock. During the follow-up period, 10 patients (9\%) underwent cardiac transplantation. Mortality

13 from any cause occurred in 9 patients (8\%), three of whom had previously undergone

14 transplantation. Details of the cause of death were available in 7 of these 9 patients

15 (heart failure $[n=3]$; VT storm $[n=1]$; VF $[n=1]$; stroke $[n=1]$; sepsis $[n=1]$ ). As

16 demonstrated in Figure 7, there was no significant difference in survival between

17 patients who were treated with $\mathrm{AAD} / \mathrm{BB}$ alone to those who underwent adjunctive VT 18 ablation $(p=0.61)$.

20 Early versus late ablation

21 We compared outcomes of the first-time combined endocardial/epicardial VT

22 ablation in patients who had $<10$ VT episodes/shocks prior to ablation (median 5 [IQR

23 3; variance 4.5; skewness 0.87], experienced over median of 3.3 years [IQR 3.8

24 variance 47.2; skewness 1.35]) to patients who had their first ablation after $>10 \mathrm{VT}$ 
1 episodes/shocks (median 15, [IQR 16; variance 180.8; skewness 1.05], experienced

2 over a median of 7.7 years [IQR 8.4; variance 28.9; skewness 0.43]). As shown in

3 Supplemental figure 3, ablation performed in patients with $<10 \mathrm{VT}$ episodes/shocks

4 was associated with improved VT-free survival ( $p=0.04)$. Of note, there were no

5 differences in AAD/BB therapy between the $>10$ and $<10 \mathrm{VT}$ episodes/shocks groups

6 (Supplemental table 2).

7

8 Amongst patients undergoing endocardial ablation alone on the other hand, we did

9 not observe differences in outcome in patients with $<10$ VT episodes/shocks (median

104 [IQR 2; variance 3.4; skewness 1.38], experienced over a median of 2.5 years [IQR

11 5.2; variance 32.3; skewness 1.83]) and patients with $>10 \mathrm{VT}$ episodes/shocks

12 (median 16 [IQR 5; variance 68.4; skewness 1.43], experienced over a median of 3.1

13 years [IQR 5.5; variance 21.3; skewness 0.91]). The results are shown in Supplemental

14 figure 4. Details of AAD/BB therapy between the $>10$ and $<10$ VT episodes/shocks

15 groups are included in Supplemental table 3.

16

\section{Discussion}

18 In this multicentre study of ARVC patients, we found that after $\geq 3$ VT episodes, adding

19 a single ablation procedure (endocardial-only ablation in the majority) to

20 antiarrhythmic drug therapy was not associated with better VT-free survival when

21 compared to continuing or escalating antiarrhythmic drug therapy. Overall, multiple

22 procedures were necessary to maintain freedom from VT. Consistent with previous

23 reports, combined endocardial/epicardial ablation demonstrated superior VT-free

24 survival when compared to endocardial-only ablation. We also found that early VT 
1 intervention with combined endocardial/epicardial ablation maybe associated with

2 improved VT-free survival. Finally, VT ablation did not have a significant impact on

3 mortality or the need for cardiac transplantation.

4

5 To our knowledge, this is the first report comparing outcomes in ARVC patients

6 undergoing VT ablation to patients treated with AAD/BB alone. While ARVC patients

7 had a high risk of recurrent VT despite antiarrhythmic drug therapy or ablation, it is

8 important to consider that a number of factors may have contributed to non-optimal

9 ablation strategies, which in turn may have influenced outcome. Firstly, only a third of

10 patients had epicardial ablation with their first procedure and only $53 \%$ of ablation

11 patients ever had an epicardial ablation. As discussed below, a combined

12 endocardial/epicardial approach is associated with improved VT-free survival.

13 Secondly, a significant proportion of patients in our cohort underwent late

14 interventions. As outlined above, earlier interventions may be associated with more

15 favourable outcomes. These considerations underscore the importance of

16 optimization of the ablation strategy to improve VT-free survival. Future studies

17 specifically comparing combined early endocardial/epicardial ablation to AAD/BB

18 therapy are necessary to fully define the impact of VT ablation in ARVC.

19

20 The outcome of VT ablation in ARVC has been investigated in multiple previous

21 studies. The reported freedom from VT following ablation in these studies is between

$2245 \%$ to $85 \%$, with variable procedure methods and follow-up periods. ${ }^{11,13-15,23,24}$ The

23 outcomes in the present study are comparable, with success rates of $63 \%$ at one year.

24 Furthermore, consistent with previous studies, we demonstrate that combined 
1 endocardial/epicardial ablation is associated with superior VT-free survival as

2 compared to endocardial ablation alone. ${ }^{11,14,23}$ It is important to note however, that

3 a number of more recent reports have suggested that in selected patients,

4 endocardial-only ablation is associated with comparable long-term outcomes to the

5 combined endocardial-epicardial approach. ${ }^{15,25}$ Furthermore, endocardial ablation

6 has been reported to be effective for elimination of epicardial VT substrates in ARVC

7 patients. ${ }^{26}$ These findings suggest that with evolving VT ablation techniques, the

8 efficacy of endocardial-only ablation may be improving.

9

10 The efficacy of different antiarrhythmic drugs in ARVC remains incompletely defined.

11 Marcus and colleagues reported that empirical therapy with amiodarone is associated

12 with superior efficacy compared to sotalol. ${ }^{27}$ In contrast, amongst patients

13 undergoing serial testing with programmed stimulation, sotalol has been reported to

14 be more effective than amiodarone in supressing ventricular arrhythmias. ${ }^{28}$ Relatively

15 little is known about the efficacy of class 1 antiarrhythmics in ARVC. The addition of

16 flecainide to sotalol/beta blockers has been reported to enhance VT-free survival. ${ }^{29}$ In

17 the present study we demonstrate that in patients with a high VT burden,

18 amiodarone and class 1 antiarrhythmic drugs are associated with a trend towards

19 improved VT-free survival when compared to sotalol. Of note, the use of amiodarone

20 in our cohort was relatively limited, which could potentially have influenced overall

21 outcome. However, future prospective studies with larger patient numbers are

22 necessary to more clearly define the relative efficacy of class 1 agents, amiodarone

23 and sotalol. 


\section{$1 \quad$ Limitations}

2 This study has the inherent limitations of a non-randomized, retrospective

3 observational study. There are also a number of potential sources of bias. Selection of

4 antiarrhythmic drugs and ablation (and ablation techniques) were left to the treating

5 physicians, with some variation among centers. The efficacy of ablation has likely

6 improved over time, which may also have a confounding effect. Acute ablation

7 outcomes were not fully defined. Specifically, while all clinical VTs were targeted in all

8 patients, data on the proportion of patients with elimination of all clinical VTs was not

9 available. Therefore, the relative efficacy of endocardial-only and combined

10 endocardial/epicardial VT ablations in the acute setting was not defined. Finally, non-

11 uniform ICD programming between participating centers is a potential confounding

12 factor that could influence device detection and therapy for recurrent arrhythmias.

13

\section{Conclusion}

15 Amongst ARVC patients with a high VT burden, mortality and survival free from

16 transplantation is not significantly different between drug- and ablation-treated

17 patients. These patients have a high risk of recurrent VT despite drug therapy and/or

18 endocardial ablation. However, optimization of the ablation strategy, including

19 epicardial ablation can be expected to improve outcomes. 
1. Basso C, Thiene G, Corrado D, Angelini A, Nava A, Valente M. Arrhythmogenic right ventricular cardiomyopathy. Dysplasia, dystrophy, or myocarditis? Circulation 1996;94:983-991.

2. Marcus Fl, McKenna WJ, Sherrill D, et al. Diagnosis of arrhythmogenic right ventricular cardiomyopathy/dysplasia: proposed modification of the Task Force Criteria. European heart journal 2010;31:806-814.

3. Sen-Chowdhry S, Morgan RD, Chambers JC, McKenna WJ. Arrhythmogenic cardiomyopathy: etiology, diagnosis, and treatment. Annual review of medicine 2010;61:233-253.

4. Polin GM, Haqqani H, Tzou W, Hutchinson MD, Garcia FC, Callans DJ, Zado ES, Marchlinski FE. Endocardial unipolar voltage mapping to identify epicardial substrate in arrhythmogenic right ventricular cardiomyopathy/dysplasia. Heart Rhythm 2011;8:76-83.

5. Thiene G, Nava A, Corrado D, Rossi L, Pennelli N. Right ventricular cardiomyopathy and sudden death in young people. N Engl J Med 1988;318:129-133.

6. Saguner AM, Duru F, Brunckhorst CB. Arrhythmogenic right ventricular cardiomyopathy: a challenging disease of the intercalated disc. Circulation 2013;128:1381-1386.

7. Wichter T, Paul M, Wollmann C, et al. Implantable cardioverter/defibrillator therapy in arrhythmogenic right ventricular cardiomyopathy: single-center experience of long-term follow-up and complications in 60 patients. Circulation 2004;109:1503-1508.

8. Corrado D, Leoni L, Link MS, et al. Implantable cardioverter-defibrillator therapy for prevention of sudden death in patients with arrhythmogenic right ventricular cardiomyopathy/dysplasia. Circulation 2003;108:3084-3091.

9. James CA, Tichnell C, Murray B, Daly A, Sears SF, Calkins H. General and disease-specific psychosocial adjustment in patients with arrhythmogenic right ventricular dysplasia/cardiomyopathy with implantable cardioverter defibrillators: a large cohort study. Circ Cardiovasc Genet 2012;5:18-24.

10. Verma A, Kilicaslan F, Schweikert RA, et al. Short- and long-term success of substrate-based mapping and ablation of ventricular tachycardia in arrhythmogenic right ventricular dysplasia. Circulation 2005;111:3209-3216.

11. Philips B, Madhavan S, James C, et al. Outcomes of catheter ablation of ventricular tachycardia in arrhythmogenic right ventricular dysplasia/cardiomyopathy. Circ Arrhythm Electrophysiol 2012;5:499-505.

12. Dalal $\mathrm{D}$, Jain $\mathrm{R}$, Tandri $\mathrm{H}$, et al. Long-term efficacy of catheter ablation of ventricular tachycardia in patients with arrhythmogenic right ventricular dysplasia/cardiomyopathy. J Am Coll Cardiol 2007;50:432-440.

13. Marchlinski FE, Zado E, Dixit S, Gerstenfeld E, Callans DJ, Hsia H, Lin D, Nayak $H$, Russo A, Pulliam W. Electroanatomic substrate and outcome of catheter ablative therapy for ventricular tachycardia in setting of right ventricular cardiomyopathy. Circulation 2004;110:2293-2298.

14. Philips B, te Riele AS, Sawant A, Kareddy V, James CA, Murray B, Tichnell C, Kassamali B, Nazarian S, Judge DP, Calkins H, Tandri H. Outcomes and 
ventricular tachycardia recurrence characteristics after epicardial ablation of ventricular tachycardia in arrhythmogenic right ventricular dysplasia/cardiomyopathy. Heart Rhythm 2015;12:716-725.

15. Santangeli P, Zado ES, Supple GE, Haqqani HM, Garcia FC, Tschabrunn CM, Callans DJ, Lin D, Dixit S, Hutchinson MD, Riley MP, Marchlinski FE. Long-Term Outcome With Catheter Ablation of Ventricular Tachycardia in Patients With Arrhythmogenic Right Ventricular Cardiomyopathy. Circ Arrhythm Electrophysiol 2015;8:1413-1421.

16. Sapp JL, Wells GA, Parkash R, et al. Ventricular Tachycardia Ablation versus Escalation of Antiarrhythmic Drugs. N Engl J Med 2016;375:111-121.

17. Marcus FI, McKenna WJ, Sherrill D, et al. Diagnosis of arrhythmogenic right ventricular cardiomyopathy/dysplasia: proposed modification of the task force criteria. Circulation 2010;121:1533-1541.

18. Sosa $E$, Scanavacca M, d'Avila A, Pilleggi F. A new technique to perform epicardial mapping in the electrophysiology laboratory. J Cardiovasc Electrophysiol 1996;7:531-536.

19. Marchlinski FE, Callans DJ, Gottlieb CD, Zado E. Linear ablation lesions for control of unmappable ventricular tachycardia in patients with ischemic and nonischemic cardiomyopathy. Circulation 2000;101:1288-1296.

20. Stevenson WG, Wilber DJ, Natale A, et al. Irrigated radiofrequency catheter ablation guided by electroanatomic mapping for recurrent ventricular tachycardia after myocardial infarction: the multicenter thermocool ventricular tachycardia ablation trial. Circulation 2008;118:2773-2782.

21. Aliot EM, Stevenson WG, Almendral-Garrote JM, et al. EHRA/HRS Expert Consensus on Catheter Ablation of Ventricular Arrhythmias: developed in a partnership with the European Heart Rhythm Association (EHRA), a Registered Branch of the European Society of Cardiology (ESC), and the Heart Rhythm Society (HRS); in collaboration with the American College of Cardiology (ACC) and the American Heart Association (AHA). Heart Rhythm 2009;6:886-933.

22. Roberts-Thomson KC, Steven D, Seiler J, Inada K, Koplan BA, Tedrow UB, Epstein LM, Stevenson WG. Coronary artery injury due to catheter ablation in adults: presentations and outcomes. Circulation 2009;120:1465-1473.

23. Bai R, Di Biase L, Shivkumar K, et al. Ablation of ventricular arrhythmias in arrhythmogenic right ventricular dysplasia/cardiomyopathy: arrhythmia-free survival after endo-epicardial substrate based mapping and ablation. Circ Arrhythm Electrophysiol 2011;4:478-485.

24. Garcia FC, Bazan V, Zado ES, Ren JF, Marchlinski FE. Epicardial substrate and outcome with epicardial ablation of ventricular tachycardia in arrhythmogenic right ventricular cardiomyopathy/dysplasia. Circulation 2009;120:366-375.

25. Mussigbrodt A, Efimova E, Knopp H, Bertagnolli L, Dagres N, Richter S, Husser D, Bollmann A, Hindricks G, Arya A. Should all patients with arrhythmogenic right ventricular dysplasia/cardiomyopathy undergo epicardial catheter ablation? J Interv Card Electrophysiol 2017;48:193-199. 


\section{Komatsu $\mathrm{Y}$, Daly $\mathrm{M}$, Sacher $\mathrm{F}$, et al. Endocardial ablation to eliminate}

epicardial arrhythmia substrate in scar-related ventricular tachycardia. J Am Coll Cardiol 2014;63:1416-1426.

27. Marcus GM, Glidden DV, Polonsky B, Zareba W, Smith LM, Cannom DS, Estes NA, 3rd, Marcus F, Scheinman MM, Multidisciplinary Study of Right Ventricular Dysplasia I. Efficacy of antiarrhythmic drugs in arrhythmogenic right ventricular cardiomyopathy: a report from the North American ARVC Registry. J Am Coll Cardiol 2009;54:609-615.

28. Wichter T, Borggrefe M, Haverkamp W, Chen X, Breithardt G. Efficacy of antiarrhythmic drugs in patients with arrhythmogenic right ventricular disease. Results in patients with inducible and noninducible ventricular tachycardia. Circulation 1992;86:29-37.

29. Ermakov S, Gerstenfeld EP, Svetlichnaya $Y$, Scheinman MM. Use of flecainide in combination antiarrhythmic therapy in patients with arrhythmogenic right ventricular cardiomyopathy. Heart Rhythm 2017;14:564-569. 
Figure 3: Outcome of therapy after 3 VT episodes/shocks. Kaplan Meier curve comparing VT-free survival between patients treated with AAD/BB-only after the $3^{\text {rd }}$ VT episode/shock (dotted line) and those treated with an adjunctive VT ablation (single ablation procedure after $3^{\text {rd }} \mathrm{VT}$ episode/shock, solid line). There was no significant difference between the two initial approaches $(p=0.46)$

Figure 2. Flow diagram demonstrating numbers of patients initially undergoing VT ablation and AAD/BB-only therapy and subsequently all patients who underwent VT ablation. Abbreviations: AAD/BB, antiarrhythmic drugs/beta blockers; ARVC, arrhythmogenic right ventricular cardiomyopathy

Figure 4: Outcome of therapy after $3 \mathrm{VT}$ episodes/shocks. Kaplan Meier curve comparing VT-free survival between individual AAD/BB after the $3^{\text {rd }} \mathrm{VT}$ episode/shock (amiodarone dash-dotted grey; sotalol dash-dotted black; class 1 drugs solid grey; beta blockers dashed black) and those treated with an adjunctive VT ablation (solid black). There were no significant differences in outcome between individual AAD/BB and ablation. Amiodarone, class 1 drugs and ablation were associated with a trend towards improved outcome when compared to sotalol (amiodarone vs. sotalol $p=0.20$; class 1 vs. sotalol $p=0.12$; ablation vs. sotalol $p=0.21$ ).

Figure 5: Outcome after last ablation procedure (following $2 \pm 1$ ablation procedures). Kaplan Meier curve demonstrating VT-free survival for all patients treated with ablation procedures

Figure 6: Outcome after last ablation procedure (following $2 \pm 1$ ablation procedures). Kaplan Meier curve comparing VT-free survival between patients treated with combined epicardial/endocardial ablation procedures (endo + epi ablation, dotted line), patients treated with endocardial-only ablation (endo ablation, solid line). Of the 40 patients in the combined endocardial/epicardial group and 35 patients in the endocardial-only ablation group, follow-up data was available in 38 and 34 patients, respectively. Combined endo + epi ablation was associated with a superior outcome compared to endocardial-only $(p=0.05)$. 
2 Figure 7: Kaplan Meier curve comparing overall survival free of death or cardiac

3 transplantation in ARVC patients treated with AAD/BB alone (dotted line) to patients

4 who additionally underwent VT ablation (solid line, $p=0.61$ ). Survival is plotted from

5 the time that patients experienced their third VT episode.

6 
Table 1. Baseline characteristics

\begin{tabular}{|c|c|c|c|}
\hline & Ablation $\mathrm{N}=32^{\#}$ & $\mathrm{AAD} / \mathrm{BB} \mathrm{N}=77^{\#}$ & P value \\
\hline Age (at first VT) & $36 \pm 13$ & $39 \pm 18$ & 0.35 \\
\hline Male (\%) & $28(88 \%)$ & $63(81 \%)$ & 0.37 \\
\hline Caucasian (\%) & 31 (97\%) & $74(96 \%)$ & 0.80 \\
\hline LVEF (\%) & $55 \pm 13$ & $52 \pm 14$ & 0.39 \\
\hline \multicolumn{4}{|c|}{$\begin{array}{l}\text { Global/regional dysfunction and } \\
\text { structural alterations* }\end{array}$} \\
\hline Major (\%) & 18 (56\%) & $48(62 \%)$ & \multirow[t]{2}{*}{0.72} \\
\hline Minor (\%) & $5(16 \%)$ & $9(12 \%)$ & \\
\hline \multicolumn{4}{|c|}{ Tissue characterization of wall* } \\
\hline Major (\%) & $2(7 \%)$ & $4(5 \%)$ & \multirow{2}{*}{0.79} \\
\hline Minor (\%) & $0(0 \%)$ & $1(1 \%)$ & \\
\hline \multicolumn{4}{|c|}{ Repolarization abnormalities* } \\
\hline Major (\%) & $18(56 \%)$ & $49(64 \%)$ & \multirow{2}{*}{0.06} \\
\hline Minor (\%) & $4(13 \%)$ & $19(40 \%)$ & \\
\hline \multicolumn{4}{|l|}{$\begin{array}{l}\text { Depolarization/conduction } \\
\text { abnormalities* }\end{array}$} \\
\hline Major (\%) & $10(31 \%)$ & $29(38 \%)$ & \multirow{2}{*}{0.14} \\
\hline Minor (\%) & $6(19 \%)$ & $22(29 \%)$ & \\
\hline \multicolumn{4}{|l|}{ Family history* } \\
\hline Major (\%) & $15(47 \%)$ & $46(60 \%)$ & \multirow{2}{*}{0.34} \\
\hline Minor (\%) & $0(0 \%)$ & $1(1 \%)$ & \\
\hline Genotype positive (\%) & $15 / 21^{9}(71 \%)$ & $38 / 52^{9}(73 \%)$ & \\
\hline PKP2 & 9 & 22 & \\
\hline DSC2 & 1 & 2 & \\
\hline DSG2 & 2 & 3 & \\
\hline DSP & 1 & 5 & \\
\hline JUP & & 1 & \\
\hline PLN & & 1 & \\
\hline TMEM43 & 2 & 4 & \\
\hline \multicolumn{4}{|l|}{ Drugs at $3^{\text {rd }} V T$ episode } \\
\hline Beta blockers & $6(19 \%)$ & $17(22 \%)$ & 0.72 \\
\hline Sotalol & $13(40 \%)$ & $25(33 \%)$ & 0.49 \\
\hline Amiodarone & $7(22 \%)$ & $8(10 \%)$ & 0.10 \\
\hline Class I & $1(3 \%)$ & $11(14 \%)$ & 0.09 \\
\hline None & $5(16 \%)$ & $16(21 \%)$ & 0.55 \\
\hline \multicolumn{4}{|l|}{ Drugs after $3^{\text {rd }} V T$ episode } \\
\hline Beta blockers & $4(13 \%)$ & $12(16 \%)$ & 0.69 \\
\hline Sotalol & $15(47 \%)$ & $35(45 \%)$ & 0.85 \\
\hline Amiodarone & $3(9 \%)$ & $20(26 \%)$ & 0.05 \\
\hline Class 1 & $2(6 \%)$ & $10(13 \%)$ & 0.29 \\
\hline None & $8(25 \%)$ & $0(0 \%)$ & 0.0001 \\
\hline
\end{tabular}

"One $\$ \mathbb{P}$ the 110 ARVC patients in the study was not treated with either AAD/BB or VT ablation ${ }^{*}$ Abnoßmalities as defined by 2010 ARVC taskforce criteria. ${ }^{17}{ }^{9}$ No. patients who underwent genot/\$ing. Abbreviations: LVEF. left ventricular ejection fraction; VT. ventricular tachycardia 